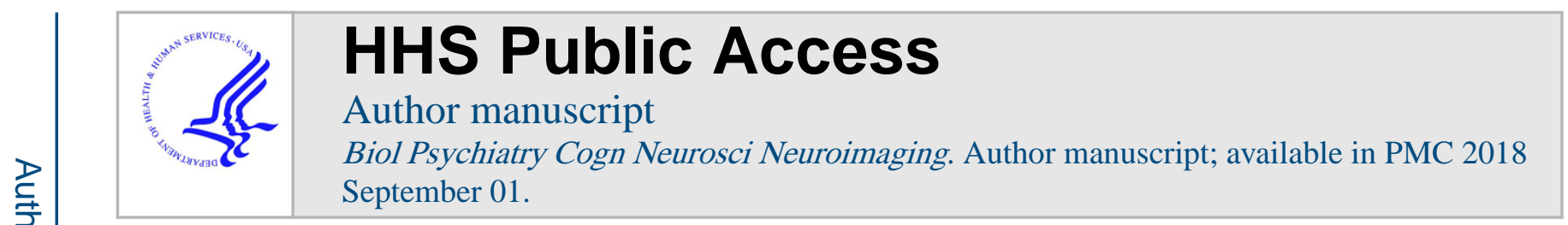

Published in final edited form as:

Biol Psychiatry Cogn Neurosci Neuroimaging. 2017 September ; 2(6): 476-486. doi:10.1016/j.bpsc. 2017.04.004.

\title{
The Default Mode Network in Autism
}

\author{
Aarthi Padmanabhan $1,{ }^{*}$, Charles J. Lynch ${ }^{4,{ }^{*}}$, Marie Schaer ${ }^{5}$, and Vinod Menon ${ }^{1,2,3}$
}

1Department of Psychiatry and Behavioral Sciences, Stanford University School of Medicine, Stanford, CA ${ }^{2}$ Program in Neuroscience, Stanford University School of Medicine, Stanford, CA ${ }^{3}$ Department of Neurology and Neurological Sciences, Stanford University School of Medicine, Stanford, CA ${ }^{4}$ Department of Psychology, Georgetown University, Washington, DC ${ }^{5}$ University of Geneva, Department of Psychiatry, Geneva, Switzerland

\begin{abstract}
Autism spectrum disorder (ASD) is characterized by deficits in social communication and interaction. Since its discovery as a major functional brain system, the default mode network (DMN) has been implicated in a number of psychiatric disorders, including ASD. Here we review converging multimodal evidence for DMN dysfunction in the context of specific components of social cognitive dysfunction in ASD: 'self-referential processing' - the ability to process social information relative to oneself and 'theory of mind' or 'mentalizing' - the ability to infer the mental states such as beliefs, intentions, and emotions of others. We show that altered functional and structural organization of the DMN, and its atypical developmental trajectory, are prominent neurobiological features of ASD. We integrate findings on atypical cytoarchitectonic organization and imbalance in excitatory-inhibitory circuits, which alter local and global brain signaling, to scrutinize putative mechanisms underlying DMN dysfunction in ASD. Our synthesis of the extant literature suggests that aberrancies in key nodes of the DMN and their dynamic functional interactions contribute to atypical integration of information about the self in relation to 'other', as well as impairments in the ability to flexibly attend to socially relevant stimuli. We conclude by highlighting open questions for future research.
\end{abstract}

\section{Keywords}

Autism; social; default mode network; theory of mind; mentalizing; self-referential processing

\footnotetext{
Address for correspondence: Vinod Menon, Ph.D. 401 Quarry Rd., Department of Psychiatry \& Behavioral Sciences Stanford University, Stanford, CA 94304-5719, menon@stanford.edu.

Equal contribution

Publisher's Disclaimer: This is a PDF file of an unedited manuscript that has been accepted for publication. As a service to our customers we are providing this early version of the manuscript. The manuscript will undergo copyediting, typesetting, and review of the resulting proof before it is published in its final citable form. Please note that during the production process errors may be discovered which could affect the content, and all legal disclaimers that apply to the journal pertain.

Financial Disclosure: All authors repot no financial interests or potential conflicts of interest
} 


\section{Autism spectrum disorder, social deficits, and the default mode network}

Autism, derived from the Greek word auto meaning "self", describes a lack of interest in social interactions with the "other". The term autism was first used by Eugen Bleuler to describe adolescents and adults with schizophrenia (1), and it became the cornerstone for Leo Kanner's characterization of infants and young children who showed a lack of interest in communicating with others and appeared to be "lost in their own narrow worlds" (2). Although these early descriptions have been influential in describing the cluster of social impairments now known to characterize autism spectrum disorder (ASD), it has become increasingly evident that self- related cognitive processing is also atypical in affected individuals (3). Understanding of "self" in the context of "other" is integral to successful social interactions (4), and it is theorized that individuals with ASD struggle with reciprocal social interaction largely due to difficulties in both self-referential cognitive processing and inferring the mental states of others (5). Perhaps it is not surprising then that the default mode network (DMN), a core brain system for processing information about the "self" and "other" $(6,7)$, has emerged as a key system underlying social dysfunction in ASD (3, 6-9).

Despite the unique functional properties of the DMN and its links to the ASD phenotype (10), few attempts have been made to synthesize the extant multimodal literature and provide a unified framework of DMN dysfunction in ASD. Here, we review converging evidence from multiple scales of brain organization that DMN dysfunction is a significant component of social impairments in ASD. We first describe the functional architecture of the DMN, focusing on aspects of social function that are known to be affected in ASD, including 'self-referential processing', which is the ability to process social information relative to oneself, and 'mentalizing' or 'theory of mind', which is the ability to infer the mental states, such as beliefs, intentions, and emotions, of others. We provide evidence for aberrant function of the DMN in ASD as it relates to deficits in these domains of social cognition. We then review functional, structural, cytoarchitectural, and neurophysiological evidence for neuronal disorganization of key nodes of the DMN in ASD. Our synthesis of the extant literature suggests that an altered developmental trajectory of structural and functional organization of the DMN is a prominent neurobiological feature of ASD. We discuss putative neuronal mechanisms underlying DMN dysfunction and highlight questions for future research.

\section{Functional neuroanatomy of the default mode network and its role in social cognition}

Over the past three decades, a number of influential studies have consistently demonstrated that a strongly intrinsically interconnected network of brain structures $(11,12)$ including the posterior cingulate cortex (PCC), precuneus, medial prefrontal cortex (mPFC), temporoparietal junction (TPJ), and hippocampus (13) (Figure 1), is attenuated during a wide range of cognitive tasks $(13,14)$. In parallel, several investigations have also uncovered that these structures, collectively named the "default mode network" (DMN) are significantly engaged during tasks involving social cognitive mental processes that are evaluative in nature (15-17), including self-referential and autobiographical processing (13- 
$15,18)$, and mentalizing and theory of mind $(13,17)$. Beyond socially relevant functions, the DMN is also associated with mind wandering and initiation of spontaneous thought processes (13) and subjective value judgments (19), but how these functions contribute to social cognition is not known. Given its central importance to the phenomenology of ASD, we focus on processes that are more directly relevant to social function. Analysis of the extant literature in neurotypical individuals reveals considerable overlap between core DMN nodes and brain regions involved in social cognition $(6,17,20-22)$, most notably the PCC, mPFC, and TPJ (Figure 2). We next summarize some of the known functional roles of these three brain regions as relevant for the present review. As one of the most highly connected regions in the brain (23), with a high baseline metabolic rate (16), the PCC is considered to be a core functional "hub". The PCC, which is situated between the marginal ramus of the cingulate sulcus and the parieto-occipital sulcus, has been implicated in both self- and otherrelevant processing, including tasks requiring autobiographical memory and imagining oneself in the future (20), and evaluating and processing others' mental states $(15,24,25)$.

The mPFC encompasses a collection of strongly interconnected, contiguous regions in the prefrontal cortex, including the medial superior frontal, orbital, and frontopolar cortices, and anterior portions of the cingulate cortex. The mPFC is associated with both monitoring of one's own mental states and the mental states of others $(15,17)$, which are thought to engage the ventral and dorsal subregions, respectively $(13,26)$.

The TPJ is situated between the inferior parietal cortex and posterior superior temporal cortex, with prominent overlap with the angular gyrus node of the DMN (27). The TPJ preferentially encodes 'other-relevant' information, including the mental states and beliefs of others. For example, transcranial magnetic stimulation to the right TPJ has been shown to disrupt a participant's ability to attribute intentions to others (28) and the ability to distinguish 'other-relevant' from 'self-relevant' information (29). The TPJ has also been linked with predicting other's behaviors during social interaction (30).

Thus, the PCC, dorsal and ventral mPFC, and TPJ, core DMN nodes, play distinct and interacting roles in monitoring of both the psychological state of self and evaluation of others.

\section{Task-based fMRI studies of atypical DMN function}

A prominent cognitive deficit of ASD is impairment in the ability to decode the mental states of self and others, and DMN dysfunction may be a critical neural signature of these deficits (31-33). The majority of task-based fMRI studies of ASD have been conducted with adults, or mixed groups of adolescents and adults, with ASD relative to age-matched neurotypical individuals. Task-related fMRI studies also focus primarily on activation in specific nodes of the social brain, including the DMN regions described above.

Studies of self-referential processing, requiring self- versus other-related judgments, demonstrate reduced activation in the PCC $(34,35)$ and $\mathrm{mPFC}(35)$ and reduced connectivity between the two regions in ASD (33). Another study, which compared the neural response in the ventral mPFC to self- vs. other-related judgments, showed preferential activation of this 
region for self-related judgments in neurotypical control, but not in adults with ASD (33).

Analysis of multivariate voxel patterns further suggests that these midline structures, and the PCC in particular, are insensitive to semantic processing of words that connote social interactions in adults with ASD. Moreover, machine learning algorithms classified individuals as autistic or control with 97\% accuracy from their fMRI neurocognitive markers in these regions (36). Together, results suggest that the PCC and mPFC exhibit aberrant patterns of self-representation in ASD.

Theory of mind and mentalizing tasks typically involve viewing images, animation, or stories, and test participants understanding of others' intentions or mental states. Overall, these studies report decreased recruitment of the TPJ and dorsal mPFC in adults with ASD (37-42). However, other studies report decreases only in relation to sexual dimorphism in ASD, with one study showing that male, but not female, adults with ASD demonstrate decreased activity in MPFC and TPJ relative to neurotypical adults (43), while a different study suggested the opposite pattern, showing that females, but not males, in the ASD group demonstrate decreased activation in the mPFC (44). Few studies have examined functional connectivity during such tasks and report decreased inter-hemispheric connectivity of the TPJ with the left lateral temporal cortex (45) and between TPJ and mPFC (46) in adults with ASD.

There have been far fewer task-based fMRI investigations in children with ASD, likely due to difficulties with task compliance and excessive head movement. When asked to either infer the face's emotional state (other-task) or to judge their own emotional response (selftask), individuals with ASD (12-31 years) show increased dorsal mPFC activation whereas neurotypical controls show age-related decreases (47). Further evidence that group differences may be age-dependent comes from a study using a similar task that found no significant differences when children and adolescents with ASD were pooled together (48). Lastly, studies using theory of mind tasks report reduced activation of dorsal mPFC, TPJ, and PCC in children (49) while other studies report increased activation in adolescents with ASD (50) relative to age- matched controls. More studies are needed to clarify the developmental profile of functional deficits in ASD.

The extant literature suggests that social impairments in ASD are linked to atypical function of the DMN. Despite considerable heterogeneity with respect to age and gender of participants and type of task, findings generally point to hypoactivation of DMN nodes in ASD, with decreased recruitment of ventral mPFC during processing of 'self-relevant' information and dorsal mPFC and TPJ during processing of 'other-relevant' information. A significant limitation is the lack of developmental studies and connectivity-based approaches. Despite the strong interconnectivity of the DMN and a large body of evidence that ASD is a disorder of network- level brain organization, it is surprising that very little is known regarding functional connectivity of the DMN during social tasks. As reviewed below, there is stronger and more consistent evidence from intrinsic connectivity studies that the overall functional organization of the DMN is aberrant in ASD. 


\section{Intrinsic functional connectivity evidence for aberrant DMN}

Intrinsic functional connectivity as measured by resting-state fMRI has been widely used to investigate the functional architecture of the DMN in $\operatorname{ASD}(9,51)$. Resting-state fMRI has been used more extensively in studies of ASD than task-related fMRI due to the relative ease of acquiring the data, especially in developmental cohorts. These studies consistently demonstrate that the DMN is among the most disrupted functional networks in ASD (10, 52). Importantly, a number of studies report that disrupted intrinsic DMN organization is associated with social deficits in children and adults with ASD (53).

The majority of intrinsic functional connectivity studies in children with ASD report increased within-network connectivity between core DMN nodes $((54,55)$, while studies in adolescents and adults report decreased connectivity (35, 56-62), and mixed age groups report both increases and decreases $(54,63-66)$. These 'inconsistencies' likely reflect developmental changes as well as heterogeneity in connectivity profiles across different nodes of the DMN (9). For example, we previously showed that children with ASD demonstrate strong hyper- connectivity between the PCC and lateral and medial temporal lobe but hypo-connectivity within DMN subregions (54) (Figure 3A). Furthermore, PCC hyper-connectivity also predicted social communication deficits in children with ASD (Figure 3B) (54). Over typical development, intrinsic functional connectivity between DMN nodes increases between childhood and adulthood (67) (Figure 4A, B). In ASD, there appears to be no consistent evidence of such increases $(61,68,69)$ in DMN connectivity with age. This pattern suggests that hyper- connected DMN links in children with ASD may become under-connected as they fail to strengthen long-range pathways with age in the same way as their neurotypical controls. Finally, cross-network connectivity also increases with age in neurotypical individuals (Figure 4C) $(70)$, but not in $\operatorname{ASD}(71,72)$ suggesting that the development of critical intra- and inter-network connections is altered in ASD.

The extant literature provides converging evidence for aberrant intrinsic DMN organization and development in ASD. Overall, the most commonly observed profile in childhood ASD has three features: (i) increased within-network connectivity in the DMN, most notably between the PCC and mPFC, (ii) reduced connectivity of DMN nodes with other functional systems outside the DMN, and (iii) increased local connectivity within DMN nodes. These patterns appear to take on a more complex profile with age involving an overall developmental shift from hyper-connectivity in childhood to hypo-connectivity in adolescence and adulthood. Such altered connectivity between DMN nodes likely underlie disturbances in self-referential and mentalizing processes, whereas reduced intrinsic connectivity between DMN and other functional systems may reflect a difficulty in adaptively switching between the DMN and networks involved in monitoring and attending to salient stimuli (35).

As reviewed above, there is now considerable evidence for aberrant functional organization of the DMN in ASD, with the strongest and most consistent findings to date emerging from analysis of intrinsic functional connectivity. Importantly, the converging evidence points to a complex and dynamic neurobiological phenotype of ASD, with both hyper and hypo connectivity profiles that shift with development. 


\section{Structural MRI evidence for atypical DMN in ASD}

Prominent structural aberrations in the DMN across several morphological metrics have been reported including cortical thickness and volume and gray matter density in ASD. Voxel-based morphometry evidence suggests altered PCC gray matter organization in children and adolescents with ASD, which was associated with symptom severity (73). Increased cortical thickness in the PCC (74) and ventral mPFC (75) (Figure 5C) has been reported in children, adolescents, and adults. Using a metric of cortical folding, or 'gyrification', we recently demonstrated that in a mixed age group of individuals with ASD, males, but not females, with ASD showed reduced gyrification in the ventral mPFC relative to neurotypical controls, suggesting a sex-specific locus of vulnerability (76) (Figure 5D). There is also evidence for shallower sulci in the TPJ of children with ASD (77) (Figure 5A), and reduced gray matter volume of the right TPJ in adults with ASD that predicted theory of mind deficits (Figure 5B) (78).

Studies measuring correlated changes in interregional cortical thickness (79) as a metric of structural network coherence report increases between the PCC and the adjoining retrosplenial cortex and the TPJ/angular gyrus in children with ASD (80). Other studies found decreased covariance between the PCC and the vmPFC in ASD, in children (80) and mixed age groups (6-50 years) (75). One study reported that both structural coherence and functional connectivity between the right TPJ and prefrontal cortex is reduced in adults with ASD (81). Future studies should use multimodal approaches to better characterize links between structure and function.

Developmentally, studies report atypical age-related structural change of DMN nodes in ASD, including accelerated thinning in bilateral PCC between childhood and adulthood (ages 7-39 years), which correlated with social deficits (82), and decelerated volume reduction with age (ages 7-29 years) in the ventral mPFC and the TPJ (83). Future studies should reconcile differences between volumetric versus cortical thickness rates of change, investigate why some nodes are impacted differently than others, and characterize the developmental trajectory of these structural changes. Another major limitation of this body of literature is that with a few notable exceptions $(69,73,78)$, the majority of studies do not show direct links with behavioral deficits. Nevertheless, together, MRI studies point to structural abnormalities of the DMN in ASD that appear to persist throughout development.

\section{Atypical white matter pathways associated with the DMN in ASD}

A number of diffusion (DTI) studies report atypical white matter connectivity in ASD (84). White matter tracts along the cingulum bundle connect the MPFC and PCC (Figure1C), and the majority of studies report decreased fractional anisotropy in the cingulum in children and adolescents with ASD (85-89), although some studies report increases (90, 91). Tracts connecting the PCC and TPJ have been challenging to measure with conventional DTI due to crossing fibers in this region (92); however, some studies have identified reduced fractional anisotropy in tracts adjacent to the TPJ in children and adolescents with ASD (87, 93). 
Over typical development, there are increases in fractional anisotropy withn the cingulum from childhood to adulthood $(94,95)$, reflecting increasing white matter connectivity and integrity with development. The cross-sectional literature that decreased fractional anisotropy in ASD may be most prominent in childhood $(96,97)$, with a decelerated rate of fractional anisotropy increases with age in young children with ASD (98), as well as mixedage groups of children and adults with ASD $(99,100)$, compared to age-matched controls.

The extant literature points to decreased white matter integrity in tracts linking DMN structures in ASD, most notably within the cingulum bundle. Furthermore, the maturation of these tracts from childhood to adulthood may be atypical in ASD. How immature development of DMN tracts contribute to impairments in their functional connectivity and consequently lead to social deficits in ASD is currently not known and is a critical avenue for future research.

\section{Atypical cellular organization of the DMN in ASD: evidence from post- mortem studies}

Although MRI studies provide critical information regarding aberrant brain function and structure at macroscopic levels, histological analysis of human postmortem brain tissue is necessary to elucidate the cellular processes that may go awry in ASD (101). Neuronal migration deficits in early brain development may be a critical component of the pathophysiology of ASD $(102,103)$. Recent post-mortem studies of brain tissue demonstrate altered laminar patterns and increased density of white matter neurons in superficial layers of PCC, but not in the fusiform gyrus (Figure 5E) (104). This selective disruption suggests that neuronal migration from the germinal zone to the cortical plate, which occurs between the $16^{\text {th }}$ and $20^{\text {th }}$ weeks of gestation, is affected in ASD (104). Additional studies with larger samples, and in other DMN and 'social' brain regions, are required to confirm whether cell migration deficits in ASD are specific to the PCC. If confirmed, it would provide strong evidence that early insults to the cellular organization of the PCC adversely impact brain development and contribute to the ASD phenotype by virtue of focused and early disruption to a core brain hub.

\section{Neurophysiological basis of DMN dysfunction in ASD}

A prominent neurobiological theory postulates that ASD is characterized by an E/I imbalance in local neural circuits that subserve sensory, social, and affective processes (105109). E/I imbalance in ASD is thought to alter local and global brain signaling and contribute to atypical fluctuations in regional fMRI signals $(64,110)$, leading to difficulties with modulating flexible and goal-directed behaviors. Thus, the DMN has emerged as a key target for E/I investigations using optogenetic studies in rodents, and there is evidence that elevated $\mathrm{E} / \mathrm{I}$ balance in the rodent $\mathrm{mPFC}$ is associated with impaired social function, an effect that was improved by increasing inhibitory function (111).

One mechanism for E/I imbalance is atypical expression of inhibitory interneurons, modulated by the neurotransmitter Gamma-Aminobutyric acid (GABA). During early embroyonic development, GABAergic interneurons are involved with regulating cell 
migration, differentiation, and synapse formation (112). Rodent models suggest that decreasing GABA transmission in the mPFC decreases sociability (113), consistent with our view that $\mathrm{E} / \mathrm{I}$ imbalance in the $\mathrm{DMN}$ can contribute to impaired social function in autism.

Molecular studies demonstrate altered signaling in inhibitory pathways that are modulated by GABAergic interneurons in ASD (114-116). Significant reductions in GABA expression have been found using MR spectroscopy (117) as well as in postmortem brain tissue of individuals with ASD (118) (Figure 5F). The co-localized pattern of deficits in cellmigration and GABA signaling in the cortex of individuals with ASD suggests that these deficits occur early in cortical development, potentially leading to persistent E/I imbalances throughout development (119-121) and altered brain connectivity in $\operatorname{ASD}(9,122,123)$. Crucially, these types of developmental aberrancies in local brain circuits are likely to influence global circuit function especially if they occur in widely connected hubs such as the PCC $(64,108,110)$. Studies using MR spectroscopy in combination with resting and task-based fMRI are needed to probe GABA and E/I imbalance related DMN dysfunction in ASD (117).

Research on the effects of GABA on social function have led to the development of specific drugs that potentiate GABA transmission as treatment for ASD symptoms (124). For example, the $\mathrm{GABA}_{\mathrm{B}}$ agonist arbaclofen has been used to improve social function in patients with Fragile X syndrome, a genetic condition that often presents with ASD symptoms (125). In a mouse model of autism, administration of benzodiazepines, which enhances GABAergic signaling, improved social and cognitive deficits (126). Whether altering DMN function using locally delivered $\mathrm{GABA}_{\mathrm{B}}$ agonists such as arbaclofen can improve social function is an important topic for future research.

\section{DMN dysfunction in the context of salience network aberrations in ASD}

Complex social behaviors involve cognitive and perceptual processes that are supported by interactions between large-scale brain systems, including the DMN (127). Notably, the DMN interfaces with two other major networks in the brain, the salience network (SN) and the central executive network (CEN) (127). Thus, the consequences of DMN dysfunction likely manifests also in its interactions with other brain systems $(10,127)$.

The triple network model of psychopathology (127) posits that atypical interactions with the SN might contribute to DMN dysfunction in ASD and contribute to a lack of engagement with socially-relevant stimuli. The $\mathrm{SN}$ is a large-scale neurocognitive network, anchored in the anterior insula and anterior cingulate cortex, and it plays a critical role in detecting salient stimuli and orienting attentional resources to them in an adaptive and goal-relevant manner (128). As such, reduced activation of the PCC and MPFC of the DMN may arise from weak mapping of salient socially-relevant cues with self-relevance and subjective value necessary for effective social function. Consistent with this view, recent evidence suggests that children with ASD exhibit intrinsic functional hyper-connectivity within the SN, CEN, and DMN (63) and other neural systems (64). This within-network intrinsic hyperconnectivity in ASD can result in "network isolation", limiting dynamic interactions between brain networks that are necessary for complex social behaviors $(35,129)$. 
Consistent with this view, a recent study demonstrated that intrinsic connectivity between the DMN, SN, and CEN predicted longitudinal improvements in adaptive behaviors in ASD (130), highlighting the significance of inter-network interactions in the pathophysiology of ASD (127).

\section{Concluding remarks}

We have sought to provide a comprehensive review of evidence pointing to aberrant DMN function in the context of mental processes that contribute to social deficits in ASD. These processes include, most prominently, mentalizing, theory of mind, and self-referential processing, which have consistently been shown to activate the core nodes of the DMN in neurotypical individuals. We have linked neural models of DMN function with specific phenotypic features of social dysfunction in ASD. We used this framing to delineate how DMN function is disrupted in children, adolescents, and adults with ASD. As we have shown, there is now substantial evidence that structural and functional disruptions to key nodes of the DMN, their connectivity with each other, and atypical patterns of connectivity with other brain regions play an important role in the symptomatology of ASD. Our synthesis of extant findings suggests that atypical integration of information about the "self" and the "other" in the DMN underlies social deficits in ASD.

Research on how the DMN changes from childhood to adulthood is providing fundamental new insights not only into the ontogeny of complex social functions in typically developing individuals, but also into components of social cognition that can go awry in ASD. It is clear that research on the DMN and its dysfunctional organization have laid the groundwork for a more sophisticated and principled approach to investigations of the complex, but interrelated, social and behavioral problems that define autism. Despite an explosive amount of research on individual brain regions and their interconnectivity, progress in our understanding of how the brain interactively processes socially relevant stimuli remains a major challenge for the neuroscience of ASD. Analysis of brain circuit dynamics offers new possibilities in this regard. The ability to flexibly switch between different modes of thought and reference frames is a critical feature of adaptive social function, and impairments in complex social processes in ASD are likely dependent on interactions between multiple large-scale brain systems. Progress in the field will depend on better characterization of DMN dysfunction in ASD and aberrant dynamic context-specific interactions with other brain networks such as the salience network.

The findings reviewed here highlight the necessity for studying heterogeneous patterns of functional and structural brain connectivity, and their inter-relationships at different stages of development. Longitudinal studies leveraging quantitative multimodal systems neuroscience approaches will play a crucial role moving forward. This includes novel computational tools, individualized atlases, wiring diagrams, and methodological developments that will propel systematic ways of thinking about atypical brain organization in ASD. Knowledge of how functional interactions between the DMN and other brain systems mature over time, and how they change mental representations of socially relevant information, will be fundamental to the study of the neurobiological basis of social interaction deficits in ASD. 


\section{Acknowledgments}

This work was supported by grants from the National Institutes of Health (HD059205, MH084164, NS086085 to VM), the Singer Foundation and the Simons Foundation (V.M), a Stanford Child Health Research Initiative Post Doctoral Fellowship (A.P), and a Swiss National Science Foundation Fellowship (158831 to M.S).

\section{References}

1. Bleuler, E. Organization and pathology of thought: Selected sources. Columbia University Press; New York, NY, US: 1951. Autistic thinking; p. 399-437.

2. Kanner L. Autistic Disturbances of Affective Contact. Nervous Child. 1943:217-250.

3. Uddin LQ. The self in autism: an emerging view from neuroimaging. Neurocase. $2011 ; 17(3)$ :2018. [PubMed: 21207316]

4. Banaji MR, Prentice DA. The self in social contexts. Annual review of psychology. 1994; 45(1): 297-332.

5. Baron-Cohen S, Leslie AM, Frith U. Does the autistic child have a "theory of mind"? Cognition. 1985; 21(1):37-46. [PubMed: 2934210]

6. Mars RB, et al. On the relationship between the "default mode network" and the "social brain". Frontiers in Human Neuroscience. 2012; 6

7. Molnar-Szakacs I, Uddin LQ. Self-processing and the default mode network: interactions with the mirror neuron system. Front Hum Neurosci. 2013; 7:571. [PubMed: 24062671]

8. Li W, Mai X, Liu C. The default mode network and social understanding of others: what do brain connectivity studies tell us. Front Hum Neurosci. 2014; 8:74. [PubMed: 24605094]

9. Uddin LQ, Supekar K, Menon V. Reconceptualizing functional brain connectivity in autism from a developmental perspective. Front Hum Neurosci. 2013; 7:458. [PubMed: 23966925]

10. Moseley RL, et al. Whole-brain functional hypoconnectivity as an endophenotype of autism in adolescents. Neuroimage Clin. 2015; 9:140-52. [PubMed: 26413477]

11. Hagmann P, et al. Mapping the structural core of human cerebral cortex. PLoS Biol. 2008; 6(7):e159. [PubMed: 18597554]

12. Buckner RL, et al. Cortical hubs revealed by intrinsic functional connectivity: mapping, assessment of stability, and relation to Alzheimer's disease. J Neurosci. 2009; 29(6):1860-73. [PubMed: 19211893]

13. Buckner RL, Andrews-Hanna JR, Schacter DL. The Brain's Default Network: Anatomy, Function, and Relevance to Disease. Annals of the New York Academy of Sciences. 2008; 1124(1):1-38. [PubMed: 18400922]

14. Greicius MD, Menon V. Default-mode activity during a passive sensory task: uncoupled from deactivation but impacting activation. J Cogn Neurosci. 2004; 16(9):1484-92. [PubMed: 15601513]

15. Gusnard DA, Raichle ME, Raichle ME. Searching for a baseline: functional imaging and the resting human brain. Nat Rev Neurosci. 2001; 2(10):685-94. [PubMed: 11584306]

16. Raichle ME, et al. A default mode of brain function. Proc Natl Acad Sci U S A. 2001; 98(2):67682. [PubMed: 11209064]

17. Schilbach L, et al. Minds at rest? Social cognition as the default mode of cognizing and its putative relationship to the "default system" of the brain. Conscious Cogn. 2008; 17(2):457-67. [PubMed: 18434197]

18. Andrews-Hanna JR, et al. Evidence for the default network's role in spontaneous cognition. J Neurophysiol. 2010; 104(1):322-35. [PubMed: 20463201]

19. Acikalin MY, Gorgolewski KJ, Poldrack RA. A Coordinate-Based Meta-Analysis of Overlaps in Regional Specialization and Functional Connectivity across Subjective Value and Default Mode Networks. Front Neurosci. 2017; 11:1. [PubMed: 28154520]

20. Spreng RN, Mar RA, Kim AS. The common neural basis of autobiographical memory, prospection, navigation, theory of mind, and the default mode: a quantitative meta-analysis. J Cogn Neurosci. 2009; 21(3):489-510. [PubMed: 18510452] 
21. Laird AR, et al. Behavioral interpretations of intrinsic connectivity networks. J Cogn Neurosci. 2011; 23(12):4022-37. [PubMed: 21671731]

22. Smith SM, et al. Correspondence of the brain's functional architecture during activation and rest. Proc Natl Acad Sci U S A. 2009; 106(31):13040-5. [PubMed: 19620724]

23. Leech R, Sharp DJ. The role of the posterior cingulate cortex in cognition and disease. Brain. 2014; 137(Pt 1):12-32. [PubMed: 23869106]

24. Schiller D, et al. A neural mechanism of first impressions. Nat Neurosci. 2009; 12(4):508-14. [PubMed: 19270690]

25. Kuzmanovic B, et al. Imaging first impressions: distinct neural processing of verbal and nonverbal social information. Neuroimage. 2012; 60(1):179-88. [PubMed: 22227133]

26. Amodio DM, Frith CD. Meeting of minds: the medial frontal cortex and social cognition. Nat Rev Neurosci. 2006; 7(4):268-77. [PubMed: 16552413]

27. Uddin LQ, et al. Dissociable connectivity within human angular gyrus and intraparietal sulcus: evidence from functional and structural connectivity. Cerebral Cortex. 2010; 20(11):2636-46. [PubMed: 20154013]

28. Costa A, et al. Prefrontal and temporo-parietal involvement in taking others' perspective: TMS evidence. Behav Neurol. 2008; 19(1-2):71-4. [PubMed: 18413921]

29. Heinisch C, Kruger MC, Brune M. Repetitive transcranial magnetic stimulation over the temporoparietal junction influences distinction of self from famous but not unfamiliar others. Behav Neurosci. 2012; 126(6):792-6. [PubMed: 23067386]

30. Carter RM, et al. A distinct role of the temporal-parietal junction in predicting socially guided decisions. Science. 2012; 337(6090):109-11. [PubMed: 22767930]

31. Happe F, Cook JL, Bird G. The Structure of Social Cognition: In(ter)dependence of Sociocognitive Processes. Annu Rev Psychol. 2016

32. Murdaugh DL, et al. Differential deactivation during mentalizing and classification of autism based on default mode network connectivity. PloS one. 2012; 7(11):e50064. [PubMed: 23185536]

33. Lombardo MV, et al. Atypical neural self-representation in autism. Brain : a journal of neurology. 2010; 133(Pt 2):611-24. [PubMed: 20008375]

34. Morita T, et al. Emotional responses associated with self-face processing in individuals with autism spectrum disorders: an fMRI study. Soc Neurosci. 2012; 7(3):223-39. [PubMed: 21936743]

35. Kennedy DP, Courchesne E. The intrinsic functional organization of the brain is altered in autism. Neuroimage. 2008; 39(4):1877-1885. [PubMed: 18083565]

36. Just MA, et al. Identifying autism from neural representations of social interactions: neurocognitive markers of autism. PLoS One. 2014; 9(12):e113879. [PubMed: 25461818]

37. Murdaugh DL, Nadendla KD, Kana RK. Differential role of temporoparietal junction and medial prefrontal cortex in causal inference in autism: an independent component analysis. Neurosci Lett. 2014; 568:50-5. [PubMed: 24695086]

38. Aoki Y, et al. Oxytocin improves behavioural and neural deficits in inferring others' social emotions in autism. Brain. 2014; 137(Pt 11):3073-86. [PubMed: 25149412]

39. Kana RK, et al. Altered Medial Frontal and Superior Temporal Response to Implicit Processing of Emotions in Autism. Autism Res. 2016; 9(1):55-66. [PubMed: 25962831]

40. von dem Hagen EA, et al. Direct Gaze Elicits Atypical Activation of the Theory-of-Mind Network in Autism Spectrum Conditions. Cerebral Cortex. 2013

41. Assaf M, et al. Mentalizing and motivation neural function during social interactions in autism spectrum disorders. Neuroimage Clin. 2013; 3:321-31. [PubMed: 24273716]

42. Pantelis PC, et al. A specific hypoactivation of right temporo-parietal junction/posterior superior temporal sulcus in response to socially awkward situations in autism. Soc Cogn Affect Neurosci. 2015; 10(10):1348-56. [PubMed: 25698698]

43. Kirkovski M, et al. Atypical Neural Activity in Males But Not Females with Autism Spectrum Disorder. J Autism Dev Disord. 2016; 46(3):954-63. [PubMed: 26520145]

44. Holt RJ, et al. 'Reading the Mind in the Eyes': an fMRI study of adolescents with autism and their siblings. Psychol Med. 2014; 44(15):3215-27. [PubMed: 25065819] 
45. Mason RA, et al. Theory of Mind disruption and recruitment of the right hemisphere during narrative comprehension in autism. Neuropsychologia. 2008; 46(1):269-80. [PubMed: 17869314]

46. Kana RK, et al. Atypical frontal-posterior synchronization of Theory of Mind regions in autism during mental state attribution. Soc Neurosci. 2009; 4(2):135-52. [PubMed: 18633829]

47. Schulte-Ruther M, et al. Age-dependent changes in the neural substrates of empathy in autism spectrum disorder. Social Cognitive and Affective Neuroscience. 2013

48. Vander Wyk BC, Hoffman F, Pelphrey KA. Equivalent neural responses in children and adolescents with and without autism during judgments of affect. Dev Cogn Neurosci. 2014; 8:12130. [PubMed: 24016745]

49. Kana RK, et al. Aberrant functioning of the theory-of-mind network in children and adolescents with autism. Mol Autism. 2015; 6:59. [PubMed: 26512314]

50. White SJ, et al. Autistic adolescents show atypical activation of the brain's mentalizing system even without a prior history of mentalizing problems. Neuropsychologia. 2014; 56:17-25. [PubMed: 24361475]

51. Hull JV, et al. Resting-State Functional Connectivity in Autism Spectrum Disorders: A Review. Front Psychiatry. 2016; 7:205. [PubMed: 28101064]

52. Glerean E, et al. Reorganization of functionally connected brain subnetworks in high-functioning autism. Hum Brain Mapp. 2016; 37(3):1066-79. [PubMed: 26686668]

53. Yerys BE, et al. Default mode network segregation and social deficits in autism spectrum disorder: Evidence from non-medicated children. Neuroimage Clin. 2015; 9:223-32. [PubMed: 26484047]

54. Lynch CJ, et al. Default mode network in childhood autism: posteromedial cortex heterogeneity and relationship with social deficits. Biol Psychiatry. 2013; 74(3):212-9. [PubMed: 23375976]

55. Uddin LQ, Supekar K, Menon V. Reconceptualizing functional brain connectivity in autism from a developmental perspective. Frontiers in Human Neuroscience. 2013; 7:458. [PubMed: 23966925]

56. Cherkassky VL, et al. Functional connectivity in a baseline resting-state network in autism. Neuroreport. 2006; 17(16):1687-1690. [PubMed: 17047454]

57. Monk CS, et al. Abnormalities of intrinsic functional connectivity in autism spectrum disorders. Neuroimage. 2009; 47(2):764-72. [PubMed: 19409498]

58. Weng SJ, et al. Alterations of resting state functional connectivity in the default network in adolescents with autism spectrum disorders. Brain Res. 2010; 1313:202-14. [PubMed: 20004180]

59. Wiggins JL, et al. Using a self-organizing map algorithm to detect age-related changes in functional connectivity during rest in autism spectrum disorders. Brain Res Brain Res Rev. 2011; 1380:187-97.

60. Assaf M, et al. Abnormal functional connectivity of default mode sub-networks in autism spectrum disorder patients. Neuroimage. 2010; 53(1):247-56. [PubMed: 20621638]

61. Washington SD, et al. Dysmaturation of the default mode network in autism. Human Brain Mapping. 2013

62. Jung M, et al. Default mode network in young male adults with autism spectrum disorder: relationship with autism spectrum traits. Mol Autism. 2014; 5:35. [PubMed: 24955232]

63. Uddin LQ, et al. Salience network-based classification and prediction of symptom severity in children with autism. JAMA Psychiatry. 2013; 70(8):869-79. [PubMed: 23803651]

64. Supekar K, et al. Brain hyperconnectivity in children with autism and its links to social deficits. Cell Rep. 2013; 5(3):738-47. [PubMed: 24210821]

65. Funakoshi Y, et al. Default mode network abnormalities in children with autism spectrum disorder detected by resting-state functional magnetic resonance imaging. J Med Invest. 2016; 63(3-4): 204-8. [PubMed: 27644559]

66. Fishman I, et al. Atypical cross talk between mentalizing and mirror neuron networks in autism spectrum disorder. JAMA Psychiatry. 2014; 71(7):751-60. [PubMed: 24740586]

67. Supekar K, et al. Development of functional and structural connectivity within the default mode network in young children. Neuroimage. 2010; 52(1):290-301. [PubMed: 20385244]

68 . Wiggins JL, et al. The impact of serotonin transporter genotype on default network connectivity in children and adolescents with autism spectrum disorders. Neuroimage Clin. 2012; 2:17-24. [PubMed: 24179754] 
69. Doyle-Thomas KA, et al. Atypical functional brain connectivity during rest in autism spectrum disorders. Ann Neurol. 2015

70. Power JD, et al. The development of human functional brain networks. Neuron. 2010; 67(5):73548. [PubMed: 20826306]

71. Cheng W, et al. Autism: reduced connectivity between cortical areas involved in face expression, theory of mind, and the sense of self. Brain. 2015; 138(Pt 5):1382-93. [PubMed: 25795704]

72. Olivito G, et al. Resting-State Functional Connectivity Changes Between Dentate Nucleus and Cortical Social Brain Regions in Autism Spectrum Disorders. Cerebellum. 2016

73. Uddin LQ, et al. Multivariate searchlight classification of structural magnetic resonance imaging in children and adolescents with autism. Biol Psychiatry. 2011; 70(9):833-41. [PubMed: 21890111]

74. Haar S, et al. Anatomical Abnormalities in Autism? Cereb Cortex. 2014

75. Valk SL, et al. Multicenter mapping of structural network alterations in autism. Hum Brain Mapp. 2015; 36(6):2364-73. [PubMed: 25727858]

76. Schaer M, et al. Sex differences in cortical volume and gyrification in autism. Mol Autism. 2015; 6:42. [PubMed: 26146534]

77. Dierker DL, et al. Analysis of cortical shape in children with simplex autism. Cereb Cortex. 2015; 25(4):1042-51. [PubMed: 24165833]

78. David N, et al. Right temporoparietal gray matter predicts accuracy of social perception in the autism spectrum. J Autism Dev Disord. 2014; 44(6):1433-46. [PubMed: 24305776]

79. Raznahan A, et al. Patterns of coordinated anatomical change in human cortical development: a longitudinal neuroimaging study of maturational coupling. Neuron. 2011; 72(5):873-84. [PubMed: 22153381]

80. Zielinski BA, et al. scMRI reveals large-scale brain network abnormalities in autism. PloS one. 2012; 7(11):e49172. [PubMed: 23185305]

81. Mueller S, et al. Convergent Findings of Altered Functional and Structural Brain Connectivity in Individuals with High Functioning Autism: A Multimodal MRI Study. PloS one. 2013; 8(6):e67329. [PubMed: 23825652]

82. Doyle-Thomas KA, et al. Effects of age and symptomatology on cortical thickness in autism spectrum disorders. Res Autism Spectr Disord. 2013; 7(1):141-150. [PubMed: 23678367]

83. Lin HY, et al. Regional brain volume differences between males with and without autism spectrum disorder are highly age-dependent. Mol Autism. 2015; 6:29. [PubMed: 26045942]

84. Ismail MM, et al. Studying Autism Spectrum Disorder with Structural and Diffusion Magnetic Resonance Imaging: A Survey. Front Hum Neurosci. 2016; 10:211. [PubMed: 27242476]

85. Walker L, et al. Diffusion tensor imaging in young children with autism: biological effects and potential confounds. Biol Psychiatry. 2012; 72(12):1043-51. [PubMed: 22906515]

86. Barnea-Goraly N, Lotspeich LJ, Reiss AL. Similar white matter aberrations in children with autism and their unaffected siblings: a diffusion tensor imaging study using tract-based spatial statistics. Arch Gen Psychiatry. 2010; 67(10):1052-60. [PubMed: 20921121]

87. Noriuchi M, et al. Altered white matter fractional anisotropy and social impairment in children with autism spectrum disorder. Brain Res. 2010; 1362:141-9. [PubMed: 20858472]

88. Jou RJ, et al. Diffusion tensor imaging in autism spectrum disorders: preliminary evidence of abnormal neural connectivity. The Australian and New Zealand journal of psychiatry. 2011; 45(2): 153-62. [PubMed: 21128874]

89. Catani M, et al. Frontal networks in adults with autism spectrum disorder. Brain. 2016; 139(Pt 2): 616-30. [PubMed: 26912520]

90. Billeci L, et al. White matter connectivity in children with autism spectrum disorders: a tract-based spatial statistics study. BMC Neurol. 2012; 12:148. [PubMed: 23194030]

91. Weinstein M, et al. Abnormal white matter integrity in young children with autism. Human Brain Mapping. 2011; 32(4):534-43. [PubMed: 21391246]

92. Greicius MD, et al. Resting-state functional connectivity reflects structural connectivity in the default mode network. Cereb Cortex. 2009; 19(1):72-8. [PubMed: 18403396]

93. Barnea-Goraly N, et al. White matter structure in autism: preliminary evidence from diffusion tensor imaging. Biol Psychiatry. 2004; 55(3):323-326. [PubMed: 14744477] 
94. Simmonds D, et al. Developmental stages and sex differences of white matter and behavioral development through adolescence: A longitudinal diffusion tensor imaging (DTI) study. Neuroimage. 2013

95. Lebel C, et al. Diffusion tensor imaging of white matter tract evolution over the lifespan. Neuroimage. 2012; 60(1):340-52. [PubMed: 22178809]

96. Ameis SH, Catani M. Altered white matter connectivity as a neural substrate for social impairment in Autism Spectrum Disorder. Cortex. 2015; 62:158-81. [PubMed: 25433958]

97. Ameis SH, et al. Impaired structural connectivity of socio-emotional circuits in autism spectrum disorders: a diffusion tensor imaging study. PloS one. 2011; 6(11):e28044. [PubMed: 22132206]

98. Ouyang M, et al. Atypical age-dependent effects of autism on white matter microstructure in children of 2-7 years. Hum Brain Mapp. 2016; 37(2):819-32. [PubMed: 26663516]

99. Ikuta $\mathrm{T}$, et al. Abnormal cingulum bundle development in autism: a probabilistic tractography study. Psychiatry Res. 2014; 221(1):63-8. [PubMed: 24231056]

100. Shukla DK, Keehn B, Muller RA. Tract-specific analyses of diffusion tensor imaging show widespread white matter compromise in autism spectrum disorder. Journal of Child Psychology and Psychiatry, and Allied Disciplines. 2011; 52(3):286-95.

101. Schumann CM, Nordahl CW. Bridging the gap between MRI and postmortem research in autism. Brain Res. 2011; 1380:175-86. [PubMed: 20869352]

102. Casanova EL, Casanova MF. Genetics studies indicate that neural induction and early neuronal maturation are disturbed in autism. Front Cell Neurosci. 2014; 8:397. [PubMed: 25477785]

103. Reiner $\mathrm{O}$, et al. Regulation of neuronal migration, an emerging topic in autism spectrum disorders. J Neurochem. 2016; 136(3):440-56. [PubMed: 26485324]

104. Oblak AL, et al. Altered posterior cingulate cortical cyctoarchitecture, but normal density of neurons and interneurons in the posterior cingulate cortex and fusiform gyrus in autism. Autism Res. 2011; 4(3):200-11. [PubMed: 21360830]

105. Testa-Silva G, et al. Hyperconnectivity and slow synapses during early development of medial prefrontal cortex in a mouse model for mental retardation and autism. Cereb Cortex. 2012; 22(6): 1333-42. [PubMed: 21856714]

106. Vattikuti S, Chow CC. A computational model for cerebral cortical dysfunction in autism spectrum disorders. Biol Psychiatry. 2010; 67(7):672-8. [PubMed: 19880095]

107. Rubenstein JL. Three hypotheses for developmental defects that may underlie some forms of autism spectrum disorder. Curr Opin Neurol. 2010; 23(2):118-23. [PubMed: 20087182]

108. Rubenstein JL, Merzenich MM. Model of autism: increased ratio of excitation/inhibition in key neural systems. Genes Brain Behav. 2003; 2(5):255-67. [PubMed: 14606691]

109. Zikopoulos B, Barbas H. Altered neural connectivity in excitatory and inhibitory cortical circuits in autism. Front Hum Neurosci. 2013; 7:609. [PubMed: 24098278]

110. Leveille C, et al. Enhanced connectivity between visual cortex and other regions of the brain in autism: a REM sleep EEG coherence study. Autism Res. 2010; 3(5):280-5. [PubMed: 20717953]

111. Yizhar O, et al. Neocortical excitation/inhibition balance in information processing and social dysfunction. Nature. 2011; 477(7363):171-8. [PubMed: 21796121]

112. Le Magueresse C, Monyer H. GABAergic interneurons shape the functional maturation of the cortex. Neuron. 2013; 77(3):388-405. [PubMed: 23395369]

113. Paine TA, Swedlow N, Swetschinski L. Decreasing GABA function within the medial prefrontal cortex or basolateral amygdala decreases sociability. Behav Brain Res. 2016; 317:542-552. [PubMed: 27732892]

114. Baroncelli L, et al. Brain plasticity and disease: a matter of inhibition. Neural Plast. 2011; 2011:286073. [PubMed: 21766040]

115. Pizzarelli R, Cherubini E. Alterations of GABAergic signaling in autism spectrum disorders. Neural Plast. 2011; 2011:297153. [PubMed: 21766041]

116. Thatcher RW, et al. Autism and EEG phase reset: deficient GABA mediated inhibition in thalamo-cortical circuits. Dev Neuropsychol. 2009; 34(6):780-800. [PubMed: 20183733]

117. Robertson CE, Ratai EM, Kanwisher N. Reduced GABAergic Action in the Autistic Brain. Curr Biol. 2016; 26(1):80-5. [PubMed: 26711497] 
118. Oblak AL, Gibbs TT, Blatt GJ. Reduced GABAA receptors and benzodiazepine binding sites in the posterior cingulate cortex and fusiform gyrus in autism. Brain Res. 2011; 1380:218-28. [PubMed: 20858465]

119. Schroer RJ, et al. Autism and maternally derived aberrations of chromosome $15 q$. Am J Med Genet. 1998; 76(4):327-36. [PubMed: 9545097]

120. Fatemi SH, et al. Expression of $\mathrm{GABA}(\mathrm{B})$ receptors is altered in brains of subjects with autism. Cerebellum. 2009; 8(1):64-9. [PubMed: 19002745]

121. Fatemi SH, et al. GABA(A) receptor downregulation in brains of subjects with autism. J Autism Dev Disord. 2009; 39(2):223-30. [PubMed: 18821008]

122. Menon V. Developmental pathways to functional brain networks: emerging principles. Trends in Cognitive Sciences. 2013; 17(12):627-640. [PubMed: 24183779]

123. Guo X, et al. Atypical developmental trajectory of local spontaneous brain activity in autism spectrum disorder. Sci Rep. 2017; 7:39822. [PubMed: 28057930]

124. Cellot G, Cherubini E. GABAergic signaling as therapeutic target for autism spectrum disorders. Front Pediatr. 2014; 2:70. [PubMed: 25072038]

125. Berry-Kravis EM, et al. Effects of STX209 (arbaclofen) on neurobehavioral function in children and adults with fragile $\mathrm{X}$ syndrome: a randomized, controlled, phase 2 trial. Sci Transl Med. 2012; 4(152):152ra127.

126. Han S, et al. Enhancement of inhibitory neurotransmission by GABAA receptors having alpha2,3-subunits ameliorates behavioral deficits in a mouse model of autism. Neuron. 2014; 81(6):1282-9. [PubMed: 24656250]

127. Menon V. Large-scale brain networks and psychopathology: a unifying triple network model. Trends Cogn Sci. 2011; 15(10):483-506. [PubMed: 21908230]

128. Seeley WW, et al. Dissociable intrinsic connectivity networks for salience processing and executive control. J Neurosci. 2007; 27(9):2349-56. [PubMed: 17329432]

129. Uddin LQ, et al. Brain State Differentiation and Behavioral Inflexibility in Autism. Cereb Cortex. 2014

130. Plitt M, et al. Resting-state functional connectivity predicts longitudinal change in autistic traits and adaptive functioning in autism. Proceedings of the National Academy of Sciences of the United States of America. 2015; 112(48):E6699-E6706. [PubMed: 26627261]

131. Shirer WR, et al. Decoding subject-driven cognitive states with whole-brain connectivity patterns. Cerebral Cortex. 2012; 22(1):158-65. [PubMed: 21616982]

132. van den Heuvel M, et al. Microstructural organization of the cingulum tract and the level of default mode functional connectivity. The Journal of neuroscience : the official journal of the Society for Neuroscience. 2008; 28(43):10844-51. [PubMed: 18945892]

133. Tao Y, et al. The Structural Connectivity Pattern of the Default Mode Network and Its Association with Memory and Anxiety. Front Neuroanat. 2015; 9:152. [PubMed: 26635544]

134. Andrews-Hanna JR, et al. Functional-anatomic fractionation of the brain's default network. Neuron. 2010; 65(4):550-62. [PubMed: 20188659]

135. Yarkoni T, et al. Large-scale automated synthesis of human functional neuroimaging data. Nature methods. 2011; 8(8):665-70. [PubMed: 21706013]

136. Fair DA, et al. The maturing architecture of the brain's default network. Proc Natl Acad Sci U S A. 2008; 105(10):4028-4032. [PubMed: 18322013] 


\section{Box 1: Questions for future research}

- When do structural aberrations of the DMN emerge during infancy?

- What is the relation between structural and functional aberrancies in the DMN, and how do they change with age?

- $\quad$ How does E/I imbalance impact DMN circuits and function in ASD?

- $\quad$ How does DMN dysfunction impact social information processing in children with ASD?

- What do intrinsic hyper- and hypo-connectivity of specific DMN pathways impact aberrant functional responses during social information processing tasks?

- What is the relation between heterogeneity in clinical profile of ASD and heterogeneity in DMN function?

- $\quad$ How does DMN organization differ in individuals with low-functioning ASD from high- functioning ASD?

- $\quad$ Are there sex differences in DMN function and could these sex differences explain the sex differences in prevalence rates and phenotypic presentation of the disorder?

- $\quad$ How does impaired judgment of social stimulus value and social distance contribute to social cognitive dysfunction in ASD? 
A
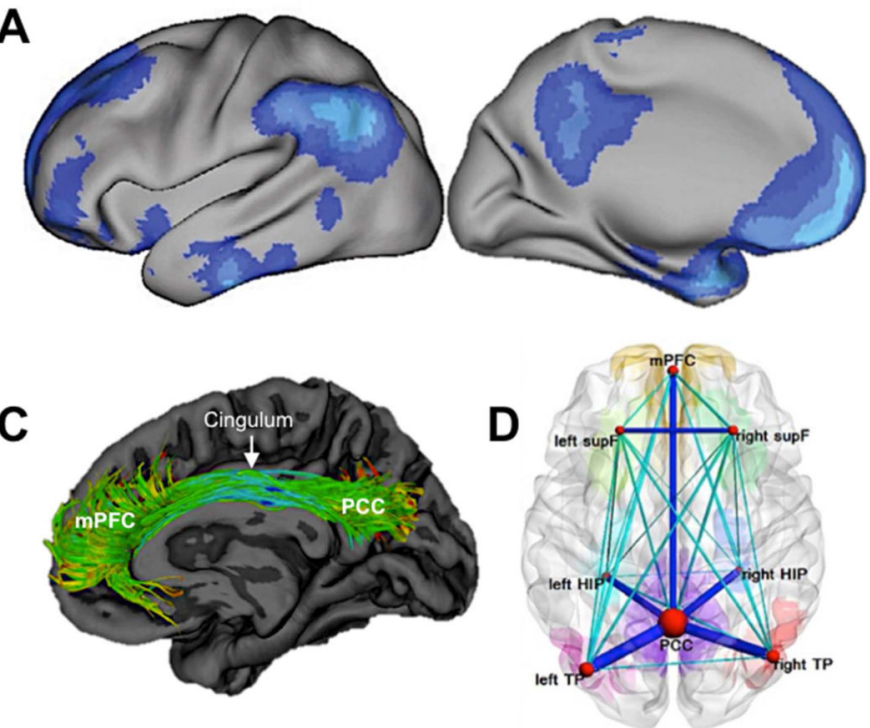
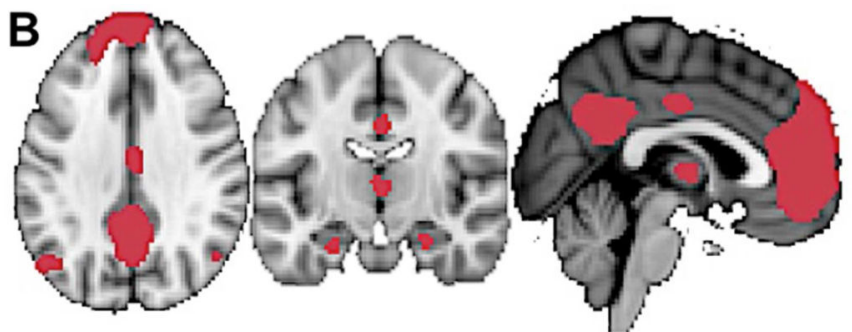

Figure 1. Functional and structural architecture of the DMN identified using multiple imaging modalities and methods

(A) Architecture of the default mode network (DMN), identified as regions of "task-induced deactivation" in the seminal meta-analysis by Shulman et al., 1999. Data derived from nine studies using $\left[{ }^{150}\right] \mathrm{H} 2 \mathrm{O}$ positron emission tomography (PET) and reproduced as a surface rendering, as in Buckner et al., 2005. (Adapted from Bucker et al., 2008 (13))

(B) DMN topology is readily identifiable using resting-state functional magnetic resonance imaging (fMRI) data, here through the use of an independent component analysis (ICA) approach. (Adapted from Shirer et al., 2012 (131))

(C) Core midline DMN nodes, the medial prefrontal cortex (mPFC) and posterior cingulate cortex (PCC) are structurally connected via a major white matter pathway, the cingulum bundle. Fibers reconstructed using diffusion tensor imaging tractography. (Adapted from van den Heuvel et al., 2008(132))

(D) The strength of structural connections amongst DMN nodes can be quantified using diffusion imaging. Edge thickness and node size represents connection strength and node degree, respectively. PCC is most strongly connected node within the DMN (Adapted from Tao et al., 2015 (133)).

(E) Spring graph illustrates the differing functional connectivity weights between DMN nodes, such that more strongly connected nodes are closer together in space and these midline "hubs" are embedded centrally within the network. (Adapted from Andrews-Hanna et al., $2010(134)$ ) 

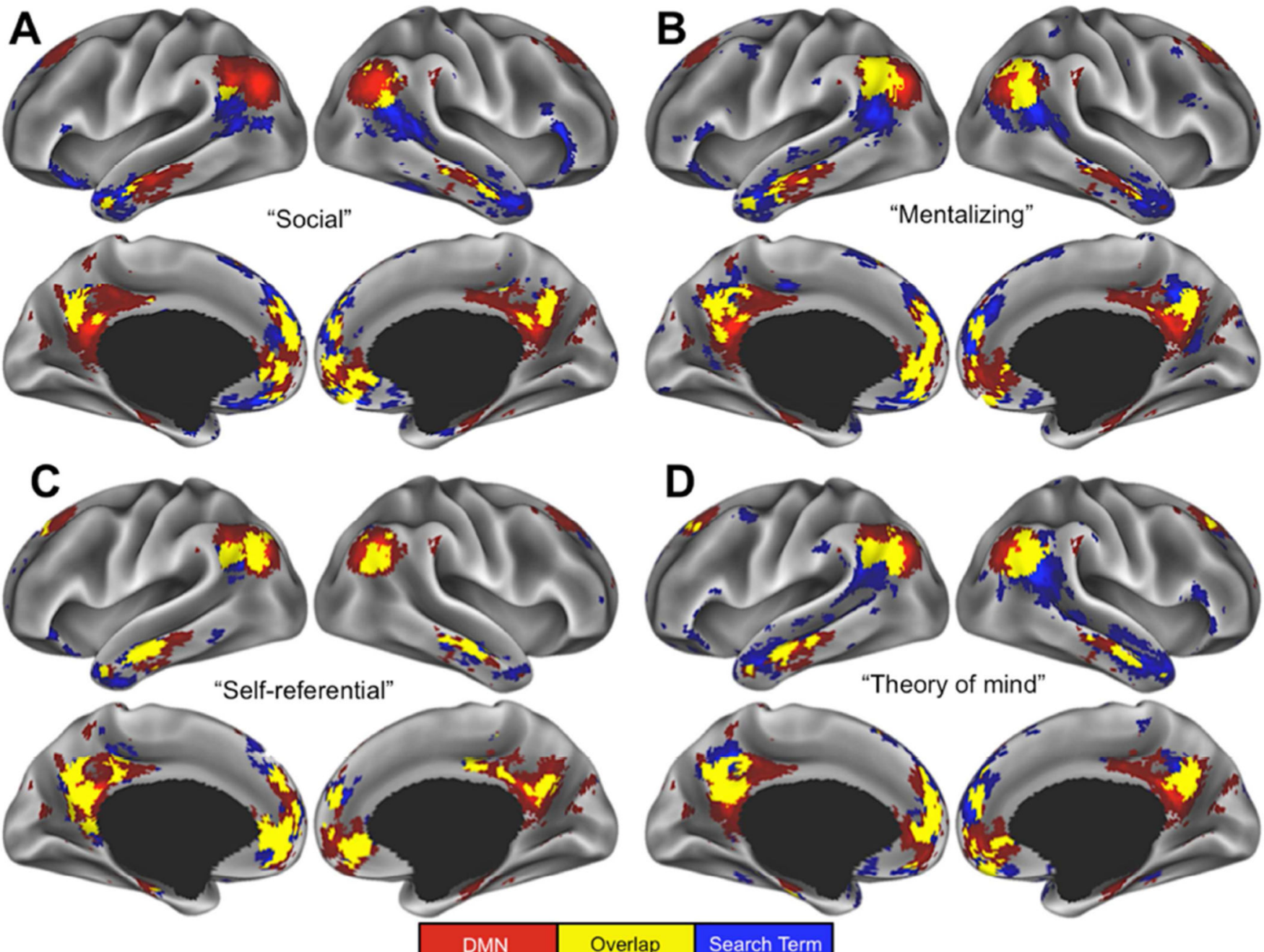

Figure 2. The DMN overlaps spatially with regions recruited by component processes of social cognition

The result of a meta-analysis using over 1200 fMRI studies in the Neurosynth database(135), using the search term "default mode" (shown in red). Regions implicated with four additional search terms, (A) "social", (B) "mentalizing", (C) "self-referential", and (D) "theory of mind", are shown in blue. The overlap between the DMN and regions recruited by these unique aspects of social cognition are shown in yellow. 

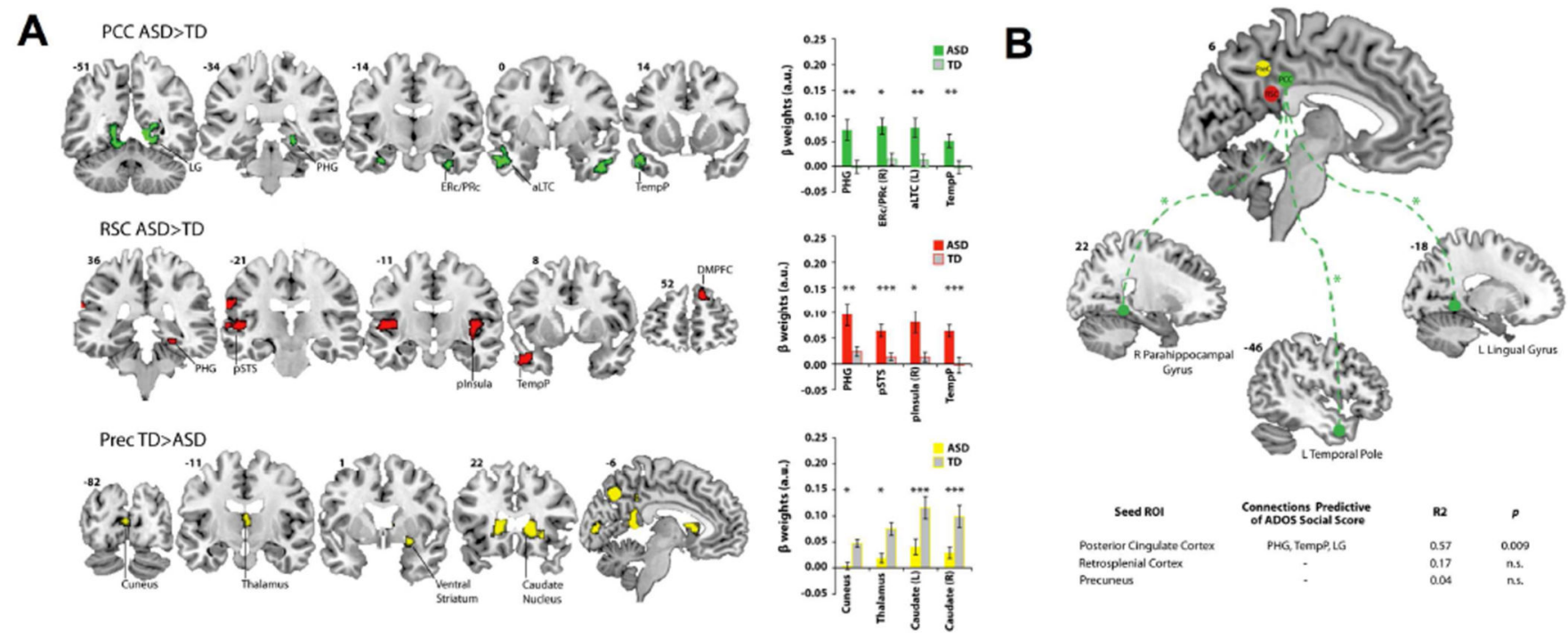

Figure 3. Hyperconnectivity with the PCC predicts social communication deficits in children with ASD

(A) Children with ASD demonstrate whole brain hyperconnectivity with PCC and retrosplenial cortex and hypoconnectivity with precuneus. ${ }^{*} \mathrm{p}<.01, * * \mathrm{p}<.005, * * * \mathrm{p}<.001$. aLTC, anterolateral temporal cortex; DMPFC, dorsomedial prefrontal cortex; ERc, entorhinal cortex; LG, lingual gyrus; PHG, parahippocampal gyrus; pInsula, posterior insular cortex; PRc, perirhinal cortex; pSTS, posterior superior temporal sulcus; TempP, temporal pole. (Adapted from Lynch et al., 2013 (54))

(B) hyperconnectivity between the PCC and target regions including the right parahippocampal gyrus, left temporal pole, and right lingual gyrus predicted social impairments as measured by the Autism Diagnostic Observation Schedule (ADOS) social subscale in children with ASD. No such significant relationships were demonstrated by the retrosplenial cortex (RSC) and precuneus (PreC). *Significant. L, left; LG, lingual gyrus; n.s., not significant; PHG, parahippocampal gyrus; R, right; TempP, temporal pole. (Adapted from Lynch et al., 2013 (54)) 

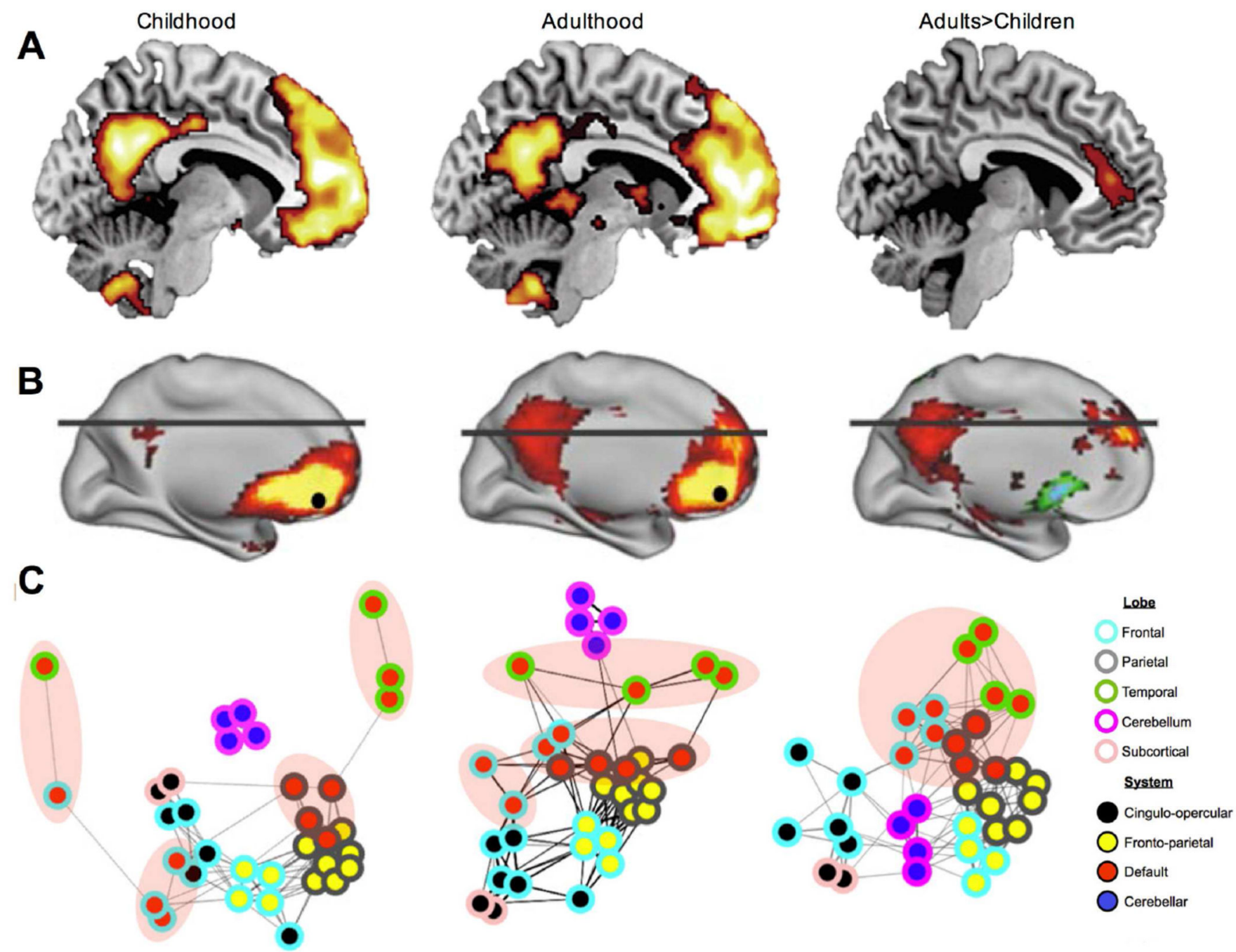

Figure 4. The DMN transitions from an immature state in childhood to a cohesive network in adulthood

(A) Independent component analysis applied to resting-state fMRI data reveals stronger mPFC functional connectivity in a group of adults relative to a group of children aged 7-9 years. (Adapted from Supekar et al., 2010 (67))

(B) Comparison of mPFC functional connectivity strength in children (left) and adults (middle) confirms greater connectivity, especially with PCC, later in development (right). (Adapted from Fair et al., 2008 (136))

(C) Connectivity between the DMN and other systems of the brain change over development as well. Spring graph representation of brain network development using resting-state fMRI during three periods of development, left $=9$ years, middle $=13$ years, right $=25$ years. Note that some DMN nodes (filled red) are isolated from one another in childhood but are more strongly integrated in adulthood. (Adapted from Power et al., 2010 (70)). 


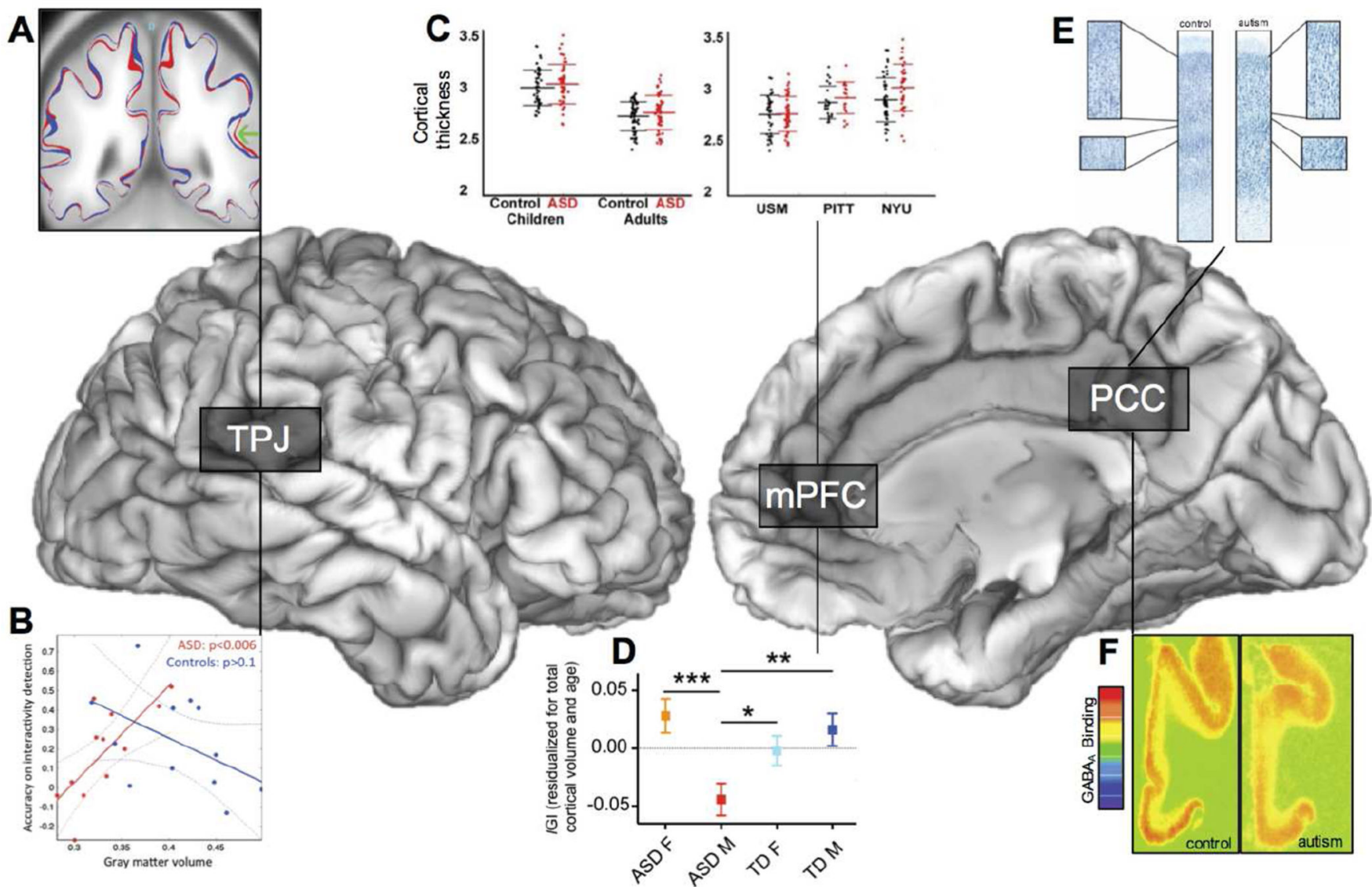

Figure 5. Structural, neurochemical, and cytoarchitectonic disorganization of key DMN nodes in ASD

(A) Right temporoparietal junction sulcus is shallower in children with ASD (red line) compared to neurotypical children (blue line). (Adapted from Dierker et al., 2015 (77)). (B) Relationship between TPJ gray matter volume and ability to assess interactions between two objects in a social motion experiment in adults with ASD (red) relative to neurotypical adult controls (blue). Note that the relationship between gray matter volume in ASD is significant, such that greater gray matter volume is associated with better performance. Adapted from David et al., 2014 (78)).

(C) Increased cortical thickness of the bilateral mPFC in children with ASD relative to adults and neurotypical children is replicable across sites of the ABIDE dataset. (Adapted from Valk et al., 2015 (75)).

(D) Differences in gyrification of the mPFC in ASD by sex. Males with ASD have reduced gyrification whereas females have increased gyrification relative neurotypical controls. (Adapted from Schaer et al., 2015 (76))

(E) Abnormal laminar pattering in post-mortem ASD PCC brain tissue (right) relative to healthy case (left). Note the general disorganization and poor differentiation between layer IV and V. (Adapted from Oblak et al., 2011 (104)) 
F) Reduced $3 \mathrm{H}$-muscimol labeled $\mathrm{GABA}_{\mathrm{A}}$ receptor binding density in the PCC in ASD (right) and neurotypical control (left) postmortem brain tissue, darker colors indicate greater receptor density. (Adapted from Oblak et al., 2011 (118)) 